\title{
Deep-ultraviolet-microelectromechanical systems stencils for high-throughput resistless patterning of mesoscopic structures
}

\author{
M. A. F. van den Boogaart ${ }^{a}$ \\ Microsystems Laboratory (LMIS1), Swiss Federal Institute of Technology (EPFL), \\ 1015 Lausanne, Switzerland \\ G. M. Kim \\ School of Mechanical Engineering, Kyungpook National University, Daegu 702-701, South Korea \\ R. Pellens and J.-P. van den Heuvel \\ ASML Special Applications, De Run 6501, 5504 DR Veldhoven, The Netherlands \\ J. Brugger \\ Microsystems Laboratory (LMIS1), Swiss Federal Institute of Technology (EPFL), \\ 1015 Lausanne, Switzerland
}

(Received 2 June 2004; accepted 9 August 2004; published 10 December 2004)

We describe a combination of 100-mm wafer scale deep-ultraviolet (DUV) exposure and a microelectromechanical systems (MEMS) process to fabricate silicon nitride membranes with submicrometer apertures to be used as miniature shadow masks or nanostencils. Apertures down to a lateral resolution of $200 \mathrm{~nm}$ were made in a 500-nm-thick membrane by DUV exposure and dry plasma etching. The membranes were released by a combination of wet silicon etching using potassium hydroxide $(\mathrm{KOH})$ and dry silicon etching using a plasma process. The millimeter-size stencils were used for single-step, local deposition of metal micro- and nano-patterns without the need for photoresist process steps. We have performed stencil deposition on full wafer scale for micro- and nano-patterns in a variety of metals (e.g. Al, Au, Ni, etc.). Dry under-etching of the nanowires resulted in free-standing cantilevered nanoelectromechanical systems (NEMS) structures with resonance frequencies in the megahertz range. The resistless method allows us to pattern micrometer and nanometer scale patterns in a single step without any further processing. It is promising for the surface processing of MEMS/NEMS devices having sensitive or fragile surfaces, such as biochips, organic polymer layers, and self-assembled monolayers. () 2004 American Vacuum Society. [DOI: 10.1116/1.1802931]

\section{INTRODUCTION}

The miniaturization of devices into the submicron range can be achieved by advanced lithography techniques using deep-ultraviolet (DUV), x-ray, electron beam, or ion beam exposure. These high-end photoresist-based methods however have major limitations if they are aimed at the processing of micro- and nano-electromechanical systems (MEMS/ NEMS) where often mechanically fragile and chemically functionalized surfaces need to be structured. Recently, the increasing demand of micron and submicron scale features for non-integrated circuit applications has given rise to a series of versatile and flexible surface patterning methods. These include scanning probe lithography, ${ }^{1}$ thermomechanical indentation of polymers by nanoimprint lithography, ${ }^{2}$ local deposition of molecules via a stamp by microcontact printing or soft-lithography, ${ }^{3,4}$ or via nanotips in dip-pen, ${ }^{5}$ and nanoscale dispensing. ${ }^{6}$ A further emerging micro/ nanopatterning method is based on a highly localized material deposition through ultra-miniature shadow-masks (nanostencils). ${ }^{7-9}$

Patterning using nanostencils allows for a wide choice of materials and surfaces to be structured as no etching pro-

\footnotetext{
${ }^{a)}$ Electronic mail: marc.vandenboogaart@epfl.ch
}

cesses need to be performed, which might damage or contaminate material layers. The use of thin membrane shadowmasks for the pattering of surfaces has been used as early as 1984. ${ }^{10}$ The stencils were fabricated by means of standard MEMS processes to obtain micron scale membrane apertures. They were applied for the patterning of micron scale features on reasonably large membranes $\left(14 \times 18 \mathrm{~mm}^{2}\right)$. Submicron features down to the $10-100 \mathrm{~nm}$ scale have been demonstrated on small areas (typically a few $10 \mu \mathrm{m}^{2}$ ). ${ }^{8}$

The main reason for the limited throughput is mainly due to the lack of a suitable high-resolution and high-throughput lithography and MEMS fabrication methods for creating nanoscale structures on large areas combined with wellcontrolled wafer-through etching of thin solid-state membranes. Serial fabrication technologies like focused ion beam $^{7,9}$ and electron beam lithography ${ }^{8,11}$ have been used to obtain apertures at nanoscale, however with limited throughput. Laser interference lithography can achieve $100 \mathrm{~nm}$ scale patterning on large areas, but is limited to periodic structures only. ${ }^{12}$

In order to overcome the limitation for the fabrication of large-area nanostencils with arbitrary apertures, we developed a $100 \mathrm{~mm}$ wafer size combined DUV/MEMS fabrication process. Using this method, aperture patterns covering 


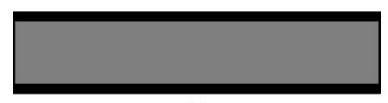

(a)

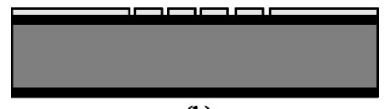

(b)

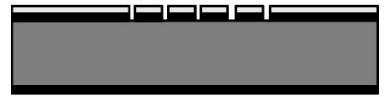

(c)

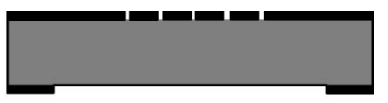

(d)

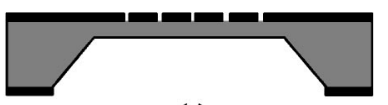

(e)

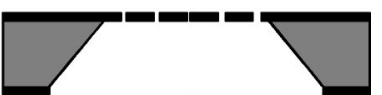

(f)
$\Longrightarrow \mathrm{Si} \rightleftharpoons \mathrm{SiN} \rightleftharpoons$ Resist

FIG. 1. Simplified schematic illustration of the fabrication process for a DUV-MEMS stencil. (a) 500-nm-thick LPCVD low-stress SiN deposition, (b) exposure and development of using DUV lithography (minimum feature size is $200 \mathrm{~nm}$ ), (c) dry etching transfer of the resist patterns into SiN, (d) membrane etch window definition by backside lithography and pattern transfer, (e) KOH etching of the bulk Si until a 10- $\mu$ m-thick Si membrane is left, and (f) final membrane releasing using dry etching, membrane size is $1 \times 1 \mathrm{~mm}^{2}$

multiple length scales, from $200 \mathrm{~nm}$ up to $300 \mu \mathrm{m}$, were defined in a thin silicon nitride layer by a wafer stepper DUV-exposure followed by dry etching.

\section{DUV BASED MICRO/NANO-STENCIL FABRICATION}

The micro/nano-stencils were fabricated using a combination of an industry standard DUV exposure and a standard MEMS processing. Figure 1 shows a schematic overview of the DUV/MEMS-based fabrication process of a full-wafer stencil. The process begins with the deposition of 500-nm-thick low-stressed silicon nitride (LS-SiN) by low pressure chemical vapor deposition (105 sccm $\mathrm{SiH}_{2} \mathrm{Cl}_{2}$, $27 \mathrm{sccm} \mathrm{NH}$, $135 \mathrm{mTorr}, 838.5{ }^{\circ} \mathrm{C}$, stress $200 \mathrm{MPa}$ tensile) on a $100 \mathrm{~mm}$ double side polished, 380- $\mu \mathrm{m}$-thick silicon (Si) wafer as illustrated in Fig. 1(a). Mesoscopic patterns which will form the membrane apertures were defined in a 500-nm-thick DUV resist (DX 5400P from AZ® Electronic Materials, Windsor, UK) followed by a $4 \times$ reduced projection exposure using a DUV wafer stepper (ASML PAS5500/ $300,248 \mathrm{~nm}$ wavelength, $10 \mathrm{~W} \mathrm{KrF}$ laser source). The patterns were developed [Fig. 1(b)] and then transferred into the 500-nm-thick LS-SiN layer by means of anisotropic etching [Fig. 1(c)]. The key process parameters for the transfer of the 500-nm-thick patterned DUV-resist into a 500-nm-thick LS-SiN layer is the selectivity of the etching agent between the DUV-resist mask layer and the SiN structural layer. In order to optimize the pattern transfer conditions, we have used a $\mathrm{C}_{2} \mathrm{~F}_{6}$ gas flow of $20 \mathrm{sccm}$ with additional $20 \mathrm{sccm}$ of $\mathrm{CH}_{4}$. Figure 2 shows a scanning electron micrograph (SEM) of the midprocess details of a typical 200-nm-scale DUVpattern after the inductively coupled plasma (ICP) transfer into the LS-SiN. This confirms the high accuracy and selectivity of the ICP transfer process at this length-scale to maintain the edge definition of the structures. The backside patterns were defined in a $1.7-\mu \mathrm{m}$-thick positive photoresist (S1818, Shipley, Coventry, UK) by means of conventional

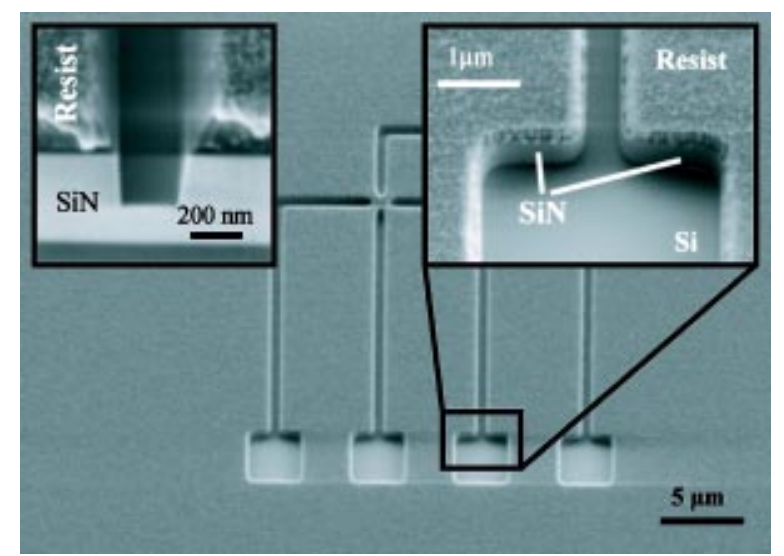

FIG. 2. Scanning electron micrograph showing a micro/nano structure after dry etch transfer into the $\mathrm{SiN}$ membrane. The left inlet shows a cross-section of the midprocess details of $200 \mathrm{~nm}$ line patterns. The right inlet shows details of an electrode pattern at submicron scale. The etch profiles demonstrate the high selectivity and process control for the transfer of the 500-nm-thick DUV resist pattern into the 500-nm-thick SiN layer.

photolithography (MA-150 aligner, SUSS MicroTec, Garching, Germany). The SiN layer on the backside was opened using ICP anisotropic etching (Alcatel 601E: $\left.\mathrm{C}_{2} \mathrm{~F}_{6} 20 \mathrm{sccm}, 20{ }^{\circ} \mathrm{C}, 1800 \mathrm{~W}\right)$. This backside patterned SiN [Fig. 1(d)] forms the etch mask for the subsequent membrane releasing [Figs. 1(e) and 1(f)].

Previous work on freestanding thin membranes often used potassium hydroxide $(\mathrm{KOH})$ etching for the release of membranes from the bulk $\mathrm{Si}$. Wet-chemical release however is often limited due to a buildup of pressure ${ }^{13}$ which can cause membrane rupture, or wet-rinsing and drying related stiction or sticking of fragile membrane features. ${ }^{14}$ Attractive capillary forces may bring mechanically compliant structures into contact with the substrate or to other parts of the membrane. After complete drying the structures remain stuck to their acquired position. In a first process we observed both wetchemical process related effects during membrane release and drying step.

As a remedy for this problem we used a combination of $\mathrm{KOH}$ and dry etching for the release of the membrane from the bulk $\mathrm{Si}$. First, $\mathrm{KOH}$ was used to etch into the bulk $\mathrm{Si}$ until an about 10-20- $\mu$ m-thick Si membrane remained. Subsequently, the remainder of the Si was etched using ICP at low temperature in order to increase the selectivity of $\mathrm{LS}-\mathrm{SiN}$ to $\mathrm{Si}$ (Alcatel 601E: $\mathrm{CF}_{6} 200 \mathrm{sccm}$, $\mathrm{O}_{2} 10-15 \mathrm{sccm},-110{ }^{\circ} \mathrm{C}, 800 \mathrm{~W}$, selectivity LS-SiN to $\mathrm{Si}$, $375: 1)$. The wet/dry process combination allows for a high yield of thin stencil membranes with compliant structures.

Figure 3(a) shows an optical picture taken from a finished full wafer $(100 \mathrm{~mm})$ containing a large variety of membrane stencils, each containing numerous mesoscopic geometrical apertures [Figs. 3(b) and 3(c)].

\section{APPLICATION OF DUV STENCIL AS FULL WAFER-SCALE SHADOW MASK}

The fabricated stencil can be used as a full wafer size shadow mask, or can be cleaved in smaller chips, and ap- 


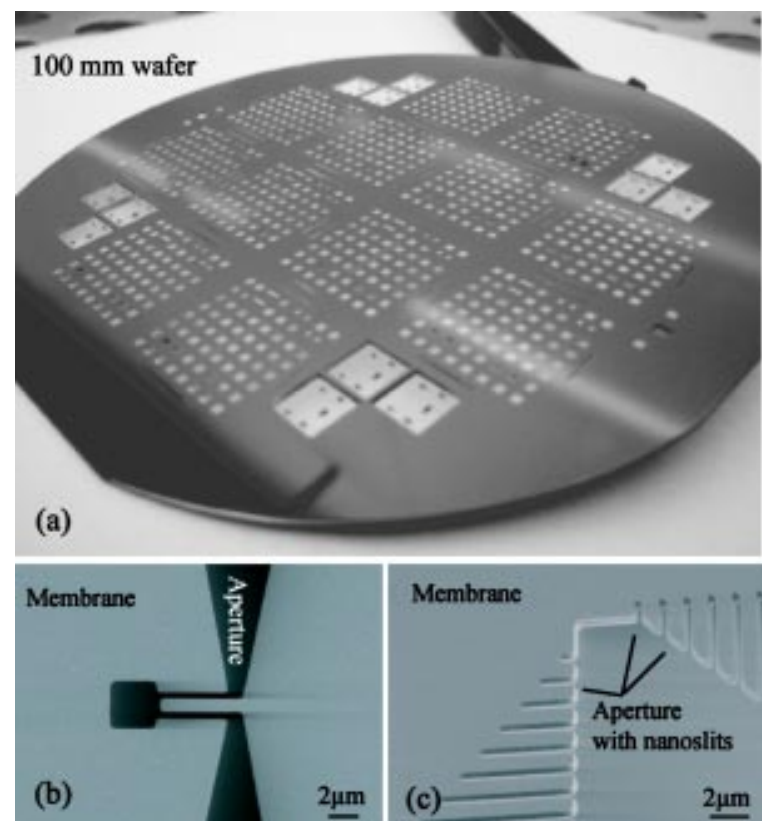

FIG. 3. (a) Optical image of a full wafer scale $(100 \mathrm{~mm})$ stencil containing various membranes, each containing numerous geometrical apertures, (b) released SiN membrane containing simultaneous micro- and nano-apertures ranging from $350 \mathrm{~nm}$ up to $30 \mu \mathrm{m}$, and (c) released SiN membrane containing micro- and nano-apertures ranging from $400 \mathrm{~nm}$ up to $300 \mu \mathrm{m}$ (not shown).

plied for local thin film deposition processes. To this extend the stencil was positioned and mechanically clamped to a substrate. Figure 4 shows a schematic illustration and SEM images of a typical stencil deposition process.

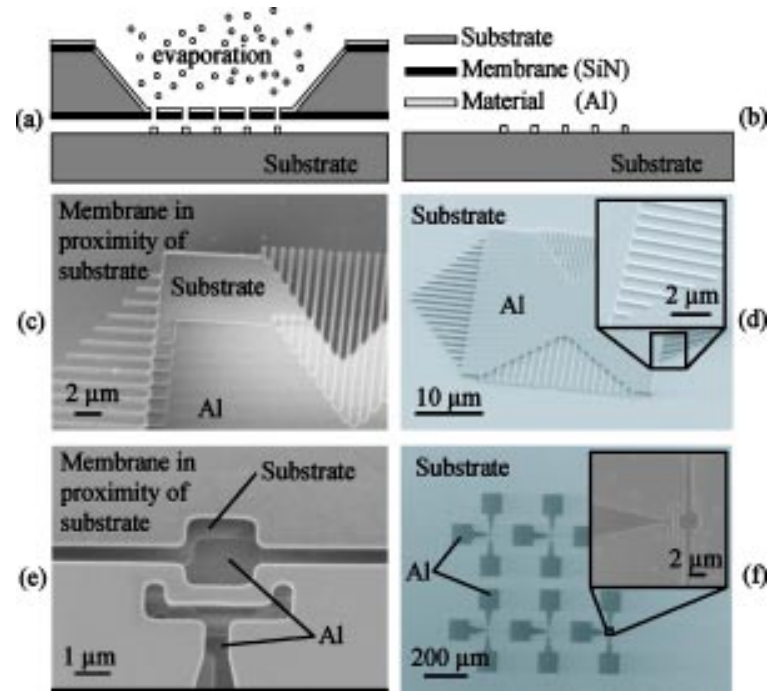

FIG. 4. Deposition process using a stencil. (a) The stencil is placed in contact or in close proximity to the substrate and a material is evaporated from a distant source and deposited through the apertures in the membrane onto the substrate, (b) the stencil is removed from the substrate, (c) and (e) the SEM-images show a stencil mask with a gap to a substrate after evaporation. The membrane in Fig. 4(c) has a gap of approximately $5 \mu \mathrm{m}$, whereas a gap of approximately $1 \mu \mathrm{m}$ is observed in Fig. 4(e), and (d) and (f) the SEMimages show the corresponding Al pattern from Figs. 4(c) and 4(e).
A physical vapor deposition evaporation system (Alcatel EVA 600) with a planetary system that can hold up to 15 wafers was used. For the stencil evaporation process the planetary rotation system was removed. The clamped stencil/ substrate was centered and placed perpendicular to the material flux at a distance of $50 \mathrm{~cm}$ from the material evaporation source. A variety of metals such as $\mathrm{Al}, \mathrm{Au}, \mathrm{Bi}, \mathrm{Cr}, \mathrm{Cu}$, $\mathrm{Ni}$, and $\mathrm{Ti}$ were locally deposited via the stencil apertures onto substrates [Fig. 4(a)]. Several substrate surfaces were successfully used such as materials that are relevant to standard semiconductor processing, e.g., $\mathrm{Si}, \mathrm{SiN}, \mathrm{SiO}_{2}$ or that are used in optoelectronic applications (GaAs). Unconventional substrates for (Bio) MEMS and NEMS have also been applied such as highly ordered pyrolytic graphite (HOPG), polydimethylsiloxane (PDMS), and epoxy based photoresist (SU-8).

Finally the stencil was removed from the substrate as shown in Fig. 4(b). Figures 4(c) and 4(e) show a stencil membrane in proximity of a substrate after evaporation of $100 \mathrm{~nm} \mathrm{Al}$. The corresponding surface structures are shown in Figs. 4(d) and 4(f) and demonstrate excellent pattern definition.

\section{RESULTS AND DISCUSSION}

A variety of structures with dimensions ranging from $200 \mathrm{~nm}$ up to $300 \mu \mathrm{m}$ were produced in large quantities and with high throughput and reproducibility on a full-wafer scale. These results show that full wafer mesoscopic stencils can be applied to a large variety of surfaces for direct rapid patterning without lithography of multiple length scale patterns on large surface areas.

The demonstrated nanostencil patterning is a practically contact-free micro- and nano-patterning method. It allows depositing small amounts of materials only at location where needed. The process can be compared to the liftoff method using a mechanical mask instead of a sacrificial photoresist layer. Using a nanostencil reduces the patterning process to a single step. Indeed, it makes other operations such as spin coating, baking, exposure, development, and etching obsolete. Hence, it can be applied on arbitrary surfaces including fragile substrates.

The deposition through stencils suffers from limitations such as the clogging of aperture, and the membrane deformation due to material induced stresses. Clogging, e.g., occurs as the evaporated material is also deposited on the membrane itself and inside the apertures. This phenomenon changes the geometrical shape of the aperture during the deposition process and leads to deformation of the deposited pattern and eventually to the complete closure of the aperture. Kölbel et al. ${ }^{15}$ demonstrated the use of self-assembled monolayers (SAM) as an antiadhesion layer. The SAMcoated SiN membranes showed a reduced aperture clogging, which improved their performance and lifetime.

A second major limitation of the membrane stencil is the stress-induced bending. The mechanically "unstable" membranes deform under influence of the deposited material due to a different thermal expansion coefficient and deposition 
related internal stress. Efforts have been made to stabilize the membrane locally by introducing some stabilization structures, ${ }^{12,13,16}$ a subject that deserves further investigations.

\section{CONCLUSIONS AND FURTHER DEVELOPMENTS}

In this article a fabrication process for the realization of full wafer stencils $(100 \mathrm{~mm})$ containing mesoscopic apertures (between $250 \mathrm{~nm}$ and $300 \mu \mathrm{m}$ ) using a combination of an industrial wafer stepper technology and an advanced MEMS process has been demonstrated. Micro- and nanoscale apertures were defined in a thin solid state $\mathrm{SiN}$ layer by means of DUV exposure and dry plasma etch process. The stencil membranes were released using a combination of wet chemical etching of the bulk silicon until approximately $10 \mu \mathrm{m}$ silicon bulk was left and subsequent dry etch releasing. This combination allows a higher yield of the membranes due to reduced risk of stiction and membrane rupture. Micro- and nano-scale structuring using this full wafer stencil allows a large choice of materials and surfaces to be nanopatterned, as no etch steps need to be applied.

We have studied nanopatterns of various materials such as $\mathrm{Al}, \mathrm{Au}, \mathrm{Bi}, \mathrm{Cr}, \mathrm{Cu}, \mathrm{Ni}$, and $\mathrm{Ti}$ for various mesoscopic experiments, on various surfaces such as $\mathrm{Si}, \mathrm{SiN}, \mathrm{SiO}_{2}, \mathrm{GaAs}$, HOPG, PDMS, and SU-8. Future work will comprise the incorporation of stabilization structures locally built into the membranes. This will allow the deposition of highly stressed materials without pattern deformation due to membrane deflection.

This fabrication process confirms the compatibility of industry standard DUV and research based MEMS technologies to achieve process selectivity and accuracy for MEMS technology at the submicrometer level.

\section{ACKNOWLEDGMENTS}

The authors are pleased to acknowledge the EPFL Center of Micro-Nano-Technology (CMI) and its entire staff as well as our colleagues at the Microsystems Laboratory for their very valuable discussions and help. This work was supported by Swiss TOP-NANO 21 through project "MELODE" (Contract No. 590.329) and by EPFL-LMIS1 start-up grant.

${ }^{1}$ K. Wilder, C. F. Quate, D. Adderton, R. Bernstein, and V. Elings, Appl. Phys. Lett. 73, 2527 (1998).

${ }^{2}$ S. Y. Chou, P. R. Krauss, and P. J. Renstrom, J. Vac. Sci. Technol. B 14, 4129 (1996).

${ }^{3}$ B. Michel, A. Bernard, A. Bietsch, E. Delamarche, M. Giessler, D. Juncker, K. Kind, J.-P. Renault, H. Rothuizen, H. Schmid, P. SchmidtWinkel, R. Stutz, and H. Wolf, IBM J. Res. Dev. 45, 697 (2001).

${ }^{4}$ A. Kumar and G. M. Whitesides, Appl. Phys. Lett. 63, 2002 (1993).

${ }^{5}$ R. D. Piner, J. Zhu, F. Xu, S. Hong, and C. A. Mirkin, Science 283, 661 (1999).

${ }^{6}$ A. Meister, S. Jeney, M. Liley, T. Akiyama, U. Staufer, N. F. de Rooij, and H. Heinzelmann, Microelectron. Eng. 67-68, 644 (2003).

${ }^{7}$ J. Koehler, M. Albrecht, C. R. Musil, and E. Bucher, Physica E (Amsterdam) 4, 196 (1999).

${ }^{8}$ M. M. Deshmukh, D. C. Ralph, M. Thomas, and J. Silcox, Appl. Phys. Lett. 75, 1631 (1999).

${ }^{9}$ G. M. Kim, M. A. F. van den Boogaart, and J. Brugger, Microelectron. Eng. 67-68, 609 (2003).

${ }^{10}$ G. Kaminsky, J. Vac. Sci. Technol. B 3, 741 (1985).

${ }^{11}$ K. Ono, H. Shimada, S.-I. Kobayashi, and Y. Ootuka, Jpn. J. Appl. Phys., Part 1 35, 2369 (1996)

${ }^{12}$ S. Kuiper, C. van Rijn, W. Nijdam, and M. Elwenspoek, J. Membr. Sci. 150, 1 (1998).

${ }^{13}$ S. Kuiper, M. de Boer, C. van Rijn, W. Nijdam, G. Krijnen, and M. Elwenspoek, J. Micromech. Microeng. 10, 171 (2000).

${ }^{14}$ R. Legtenberg, H. A. C. Tilmans, J. Elders, and M. Elwenspoek, Sens. Actuators, A 43, 230 (1994)

${ }^{15}$ M. Kölbel, R. W. Tjerkstra, K. Gyuman, J. Brugger, C. van Rijn, W. Nijdam, J. Huskens, and D. N. Reinhoudt, Adv. Funct. Mater. 13, 219 (2003).

${ }^{16}$ R. Hammer, J. Vac. Sci. Technol. 14, 1208 (1977). 